\title{
Virgilio Ducceschi y el primer laboratorio de psicología experimental en Córdoba, Argentina (1907)
}

\section{Virgilio Ducceschi and the first laboratory of experimental psychology in Córdoba, Argentine (1907)}

\section{Virgilio Ducceschi e o primeiro laboratório de psicologia experimental em Córdoba, Argentina ( 1907)}

\section{Fernando J osé Ferrari*}

Universidad Nacional de Córdoba - UNC, Córdoba, Argentina

\begin{abstract}
RESUMO
Los primeros historiadores de la psicología en Argentina y Latinoamérica han mencionado lacónicamente la presencia de Virgilio Ducceschi en el mapa de los iniciadores de la psicología experimental. Durante muchos años se perdió el rastro de este médico fisiólogo que sentaría las bases de la psicología experimental en Córdoba, Argentina. Este trabajo expone los principales escritos de este pionero de la psicología experimental Latinoamericana y lo sitúa dentro de una trama histórica que lo eleva a la par de las figuras más relevantes de la psicología Argentina y Latinoamericana: Horacio Piñero, Víctor Mercante, Cristofredo Jackob, José Ingenieros, entre otros. El trabajo pionero de Ducceschi en Latinoamérica, en Córdoba más precisamente, implicó la enseñanza de la psicología en la cátedra de psicología experimental que él crease en 1907, así como también la creación de un nuevo aparato para experimentos psicológicos: el registrador mental (1910). La cátedra de fisiología, en la que Ducceschi comenzó a desempeñarse a partir de 1906, fue creada por el Dr. De Grandis, el mismo que orientó la creación del célebre laboratorio de Horacio Piñero en Buenos Aires.
\end{abstract}

Palabras clave: Psicología Experimental, Córdoba, Argentina, Virgilio Ducceschi.

\section{ABSTRACT}

The first historians of Latin American psychology, laconically mentioned the presence of Virgilio Ducceschi on the map of the founders of experimental psychology in Latin America. For many years we lost track of this physiologist that founded the first experimental psychology in Cordoba, Argentina. This paper outlines the major writings of this pioneer of experimental psychology and offers the historical framework for understand his work. Virgilio Ducceschi, rises alongside the most relevant figures of Argentina and Latin American psychology, Horacio Piñero, Victor Merchant Cristofredo Jakob, José Ingenieros, among others. Ducceschi's pioneering work in Latin America, and in Córdoba, Argentine, involve the teaching of psychology in the department of experimental psychology created in 1907, 
as well as the creation of a new apparatus for psychological experiments: the mental registrator (1910). The chair of physiology at the start Ducceschi perform that from 1906, will be created by Dr. De Grandis, who guided the creation of the famous Horacio Piñero's laboratory in Buenos Aires.

Keywords: Experimental Psychology, Córdoba, Argentina, Virgilio Ducceschi.

\begin{abstract}
RESUMO
Os primeiros historiadores da Psicologia latino-americana têm mencionado muito laconicamente a presença de Vigilio Ducceschi entre os iniciadores da Psicologia Experimental em Latino-américa. Por muitos anos perdeu-se o rasto de este médico fisiologista que sentaria as bases da Psicologia Experimental em Córdoba, Argentina. O presente artigo expõe os principais escritos de este pioneiro da Psicologia Experimental latino-americana e situa ele numa trama histórica que o coloca ao lado das figuras mais relevantes da Psicologia argentina y latino-americana: Horacio Piñero, Victor Mercante, Cristofredo Jackob, José Ingenieros, entre outros. O trabalho precursor de Ducceschi em Latino-américa, e em Córdoba, Argentina, envolveu o ensino de Psicologia na cátedra de Psicologia Experimental que ele criou a partir de 1907, assim como a criação de um novo aparelho de experimentação psicológica: o registrador mental (1907). A cátedra de fisiologia na qual Ducceschi começará a se desempenhar desde 1906, será criada pelo Dr. De Grandis, o mesmo que orientaria a criação do célebre laboratório de Horacio Piñero em Buenos Aires.
\end{abstract}

Palavras-chave: Psicologia Experimental, Córdoba, Argentina, Virgilio Ducceschi.

\title{
1 Introducción
}

Nuestro trabajo muestra la existencia de un temprano laboratorio de Psicología Experimental en la ciudad de Córdoba, Argentina, a partir de 1907, dirigido por el italiano Virgilio Ducceschi. La figura y la obra de este médico fueron apenas mencionadas por muy pocos historiadores de psicología (GOTTHELD, 1969a y 1969b; PAPINI, M., 1976; INGENIEROS, J., 1910, VEZZETTI, 1996b.). Con el pasar de los años, los más importantes historiadores de Psicología en Argentina y Latinoamérica(ARDILA, R., 1986, 1998, 1989; KLAPPENBACH, H., 1999, 2000, 2004, 2006a,2006b, 2009; KLAPPENBACH, H. y PAVESI, P., 1994; ROSSI, L., 1997, 2005, 2007, 2008; VEZZETTI, H., 1985, 1996a , 1996b, 1996c, 1997,1998; SCHERMAN, P., 2009; PIÑEDA, 2004, 2005, 2007; TALAK, A., 1999, 2000a y 2000b.) parecen perder la pista de este pionero de la Psicología argentina y latinoamericana. Tras un intenso trabajo de archivo en la Facultad de Ciencias Médicas de la Universidad Nacional de Córdoba, hemos podido dar inicialmente con el texto de Marsal (1973) que brinda importantes datos históricos sobre las experiencias que Ducceschi realizase en Córdoba. También hemos relevado documentación y bibliografía específica en torno de la Psicología Experimental, la Psicofisiología, así como también sobre el proceso de conformación del laboratorio de 
fisiología experimental. El encuentro con la obra de Ducceschi en Córdoba abre una importante línea de investigación y brinda a la historia de la Psicología latinoamericana un nuevo pionero en los pasos previos a la constitución disciplinar.

Nos centraremos fundamentalmente en mostrar los aspectos socioinstitucionales que permitieron los desarrollos del fisiólogo italiano. Para ello situaremos sus aportes en el marco de las primeras prácticas y discursos psicológicos en Argentina. Luego relataremos el proceso de constitución de la cátedra de fisiología experimental, ámbito en donde se implantan las prácticas del laboratorio de Psicología Experimental de Ducceschi.

Posteriormente mostraremos algunas líneas de investigación que desarrolló, mediante un breve recorrido por su obra escrita. Actualmente hemos podido recopilar varios textos de Virgilio Ducceschi, respecto de sus producciones y publicaciones realizadas en el período en que cumple sus funciones en la cátedra de Fisiología. Solo mencionaremos en este trabajo los textos de mayor relevancia respecto de sus experiencias en Psicología y Psicofisiología experimentales. No obstante, el análisis discursivo de la obra escrita de Ducceschi constituye el objeto de futuras indagaciones.

\section{Inicios de la Psicología Experimental en Argentina}

Para poder situar tanto al laboratorio de Psicología Experimental como la cátedra libre de psicología dirigida por Ducceschi, recordaremos los principales hitos de práctica experimental y docente en Argentina.

Este período, caracterizado por una Psicología Experimental, clínica y social (KLAPPENBACH, 2006b, pp. 118-119.), se extendió de 1895 a 1916. La Psicología, que inundaba los estudios psiquiátricos, criminológicos, educativos y sociológicos, estaba enmarcada en dos grandes corrientes: la italiana, a partir de las teorías lombrossianas, y la francesa, a partir del degeneracionismo de Morel, retomado luego por Magnan. Dentro de las influencias francesas, Janet, Ribot y Grasset eran las personalidades más destacadas.

Tal como lo menciona Ardila (1969), Argentina fue la cuna de la Psicología en Latinoamérica. El mismo autor relata la existencia del primer laboratorio de Psicología Experimental fundado en América Latina, realizado por Horacio Piñero en 1899, en el Colegio Nacional Central. Previo a la experiencia de Piñero, Víctor Mercante funda en 1891, en la ciudad de San Juan, un laboratorio de Psicofisiología (GOTTHELD, R., 1969b, p. 148.). Los desarrollos en psicología experimental estuvieron orientados a complementar la actividad de cátedra, es decir, a la divulgación y la enseñanza (KLAPPENBACH 2006 b, p. 119.). Horacio Piñero, siendo titular de la cátedra de 
Fisiología en Medicina, sostenía al laboratorio experimental en la Facultad de Filosofía y Letras. De hecho Klappenbach (1997, p. 137138) delimita cuatro características del uso de la experimentación por parte de Piñero: i) el laboratorio ligado a una instancia pedagógica; ii) se distancia del modelo alemán en donde el laboratorio buscaba fundamentalmente la investigación original; iii) el hincapié en la clínica psicopatológica, en donde se puede detectar la concepción de Claude Bernard de la patología como experimentación natural; y iv) una enseñanza enmarcada por el programa de Theodule Ribot, en donde la Psicología abarcaba temas complejos desde la Psicofisiología de los sentidos hasta los problemas del juicio, la afectividad y la conciencia. En Buenos Aires, el Curso de Psicología comienza a dictarse en 1895, en la Facultad de Derecho y Ciencias Sociales, y luego pasaría a la Facultad de Filosofía y Letras, en 1996, bajo la dirección de Rodolfo Rivarola. Unos años después, el curso es dirigido por Horacio Piñero (1902) (KLAPPENBACH, A. H., 1997, p. 104). Es en 1907 que se aprueba la inclusión de un segundo Curso de Psicología a pedido del mismo Piñero y de Alicia Moreau. Lo destacable del caso es que los cursos de Psicología se desprendieron inicialmente de la Facultad de Filosofía y, de hecho, parte de los contenidos estaban íntimamente ligados a planteos filosóficos, en particular los del segundo curso. En este sentido si el primer curso de Psicología se dedicaba a la fisiología del sistema nervioso, el segundo curso estaría dedicado a una "psicología filosófica metafísica o pura" (KLAPPENBACH, 1997, p. 139.). De este modo la práctica experimental encontraba su limitación al abordar temas de mayor complejidad que podían ser abordados por una postura filosófica.

En Córdoba, como veremos, la cátedra libre de psicología, parece haber tenido una breve trayectoria en la Facultad de Derecho. Pero su radicación institucional será, a partir de 1906, en la Facultad de Medicina, en donde se despliega el laboratorio de Psicología Experimental.

Esta apretada síntesis nos permite ubicar las producciones de Ducceschi en Córdoba, quién se ocupa de la articulación tanto de la investigación como de la docencia:

Virgilio Ducceschi tuvo la oportunidad de desarrollar en Córdoba la organización del Laboratorio de Fisiología, que había iniciado el Prof. Valentín Grandis. I gualmente fundó las cátedras de Psicología Experimental y la de Química Biológica en la Facultad de Ciencias Médicas. (MARSAL, 1973, p. 15.). (Resaltado del autor de este artículo).

Como mostraremos en el apartado en que trataremos brevemente su obra publicada, los desarrollos del italiano se diferenciarán del tipo de aproximación que se realizó en Buenos Aires. Pero antes mostraremos el proceso socioinstitucional de constitución del espacio 
que recibirá a las primeras prácticas de Psicología Experimental en Córdoba.

\section{La cátedra de Fisiología Experimental en Córdoba}

Según nuestra investigación, podemos aseverar que el espacio que la Psicología Experimental y la Psicofisiología tuvo en Córdoba fue la Facultad de Ciencias Médicas, particularmente, la cátedra de Fisiología. En la organización de la misma tendría gran injerencia Valentín De Grandis, quién a su vez sería quien realiza la recomendación de la figura de Virgilio Ducceschi para que lo suceda.

Quien organizase la Facultad de Medicina de Córdoba, su primer decano, Dr. Hendrik Weyenberg, dictó inicialmente las materias de Anatomía, Histología y Fisiología. Pero a poco de andar, se notó la dificultad de sostener tantas materias en manos de un solo profesional, por lo que se recurrió a profesores que dictaban teóricamente la asignatura y solo ocasionalmente experiencias prácticas: Dr. Juan Bautista Gil, Dr. José A. Ortiz y Dr. Herrera, Dr. José M. Escalera, Dr. Gil R. Smith, Dr. Jerónimo del Barco (interino) y Dr. Carlos Díaz (MARSAL, A. 1973, p. 3.).

En medio de la ola inmigratoria, el Gobierno argentino contrató, en varias oportunidades, a fisiólogos italianos, más que a profesionales alemanes (MONTSERRAT, M. y ANDERMANN, J., 2000.). En Buenos Aires, Valentín De Grandis había sido el primer fisiólogo extranjero contratado. Valentín De Grandis nació en Boves (Provincia de Cuneo, Italia) el 14 de mayo de 1862. Estudió medicina en la Universidad de Turín. Allí junto a Mosso, estudió fisiología y, en particular, realizó estudios sobre el "mal de altura", estudios que sin duda tomaría Ducceschi, pues realizó experiencias en relación a la misma temática en Argentina. Luego se mudaría a Firenze, donde se formaría con el prestigioso fisiólogo Luigi Luciani. Entre 1890 y 1892 se formaría con Ludwig en Leipzig.tras lo cual sería adscripto a la Clínica psiquiátrica con Lombroso. Tras ser recomendado por el Prof. Luciani ante un pedido del ministro argentino en Roma (1899), viajó a Buenos Aires, donde permanecería 6 años. En 1897 la Universidad Nacional de Buenos Aires reorganizó la enseñanza de la Fisiología al morir el Prof. Astigueta. Grandis arriba y se desempeña como jefe de trabajos prácticos de Fisiología Experimental, al mismo tiempo que se designa como profesor suplente a Horacio Piñero, con el que en 1902 publican los Apuntes del curso de Fisiología (MARSAL, A.. 1973.). En esos años trabajaba sobre la percepción táctil. Finalmente, el italiano fue exonerado en 1903:

No parece que en el Laboratorio de Fisiología, en 1903, reinase la armonía entre sus miembros y aun cuando los 
motivos no aparecen en los archivos, no es difícil suponer que el profesor titular tendría dificultades. El 30 de junio el ayudante Sr. Carlos Mainini presentó al Decano Dr. Uballes, una enérgica nota con su renuncia, en la cual se mencionan (Archivo Facultad de Medicina Buenos Aires) acusaciones del Dr. Coronado y resultó una posición difícil para el $\mathrm{Dr}$. Grandis, que quedó mal con los dos actores principales del incidente, la Academia nombró una Comisión formada por los Dres. E. del Area, R. Herrera Vegas y Chaves, que entrevistaron al Dr. Coronado, quien declaró que era incompatible la presencia de Mainini en el Laboratorio. Con "respecto al Dr. Grandis, dijo que tiene competencia y cumple bien con los deberes de su puesto, pero no cree que hoy sea indispensable su presencia para la buena marcha del Laboratorio de Fisiología"(23 de julio de 1903). El Dr. Grandis solicitó el 22 de octubre de 1903 licencia para viajar a Europa en las próximas vacaciones. El mismo día, el Dr. Uballes le hace conocer que la Facultad, por motivos económicos, ha resuello suprimir el cargo, desde el 31 de diciembre de 1903 y le concede la licencia, sin goce de sueldo. (MARSAL A., 1973, p. 8.)

...al parecer el motivo real radicó en que Horacio Piñero, "consejero áulico de todos los decanos" y titular de la cátedra de fisiología entre 1904 y 1919, no deseaba contar en el laboratorio con alguien que poseía una indiscutible autoridad científica superior a la suya y con quien había sido, en cierto modo, su maestro"(BUCH A. en MONTSERRAT, M.; ANDERMANN, J. 2000, p. 21.).

Antes de regresar a su país, pasó por Córdoba y dirigió el Laboratorio de Fisiología en esa Universidad. Luego fue reemplazado por Virgilio Ducceschi. En el archivo de la Facultad de Ciencias Médicas de la Universidad Nacional de Córdoba (UNC) pudimos encontrar:

Contratos con profesores extranjeros. El 3 de mayo de 1904 el Sr. Decano de (ilegible) que ha nombrado interinamente al Dr. De Grandis, jefe de Trabajos prácticos de fisiología experimental, de reciente creación."(...) allí consta que el Dr. Virgilio Ducceschi, obtuvo su primer contrato en 1907 según decreto del P.E. por dos años, renovándoselo por dos años más. Tras o cual en 1910, se le dio una prorroga que dura hasta el 15 de Septiembre de 1914. Renovando prorroga nuevamente por cuatro años más, hasta 1919. (Libro de Anotaciones de Secretaría - Memorándum (1910-1940) Archivo de la Facultad de Medicina Universidad Nacional de Córdoba).

(BUCH A. en MONTSERRAT, M.; ANDERMANN, J. 2000), realiza una interpretación de la presencia de fisiólogos extranjeros, en su mayoría italianos, que parece haber tenido ciertas características:

El tipo de intervención que tuvieron puede ser comprendida por quien fue peor tratado, el menos interesado en 
cuestiones propias de la Argentina y quien tal vez mayor importancia tuvo: Valentín De Grandis. La precaria formación que pudiera tener Horacio Piñero fue un resultado, en buena medida, de la presencia del italiano en la Facultad de Medicina de la UBA entre 1899 y 1902. Lo mismo ocurrió con Lasege, Mario Camís y Ducceschi: solo podían dar los rudimentos de una enseñanza que se expresaron respectivamente en los nombres de Leopoldo Giusti, Guido Paccella y David Barilari. Los dos primeros continuarían su formación y trayectoria asociados de manera directa o indirecta con Bernardo Houssay. El último sería borrado de la historia de la fisiología en la década del 30 por el viento del progreso. (BUCH A. en MONTSERRAT, M.; ANDERMANN, J. 2000, p. 28.)

La experiencia de De Grandis en Córdoba, comenzó con la organización del Laboratorio de Fisiología Experimental. Según relata Marsal (1973) el laboratorio estuvo al nivel de los principales laboratorios sudamericanos. Y el 22 de febrero de 1904, Grandis envía desde Buenos Aires, una carta al rector Ortiz y Herrera, indicando que ese mismo día se embarca para Europa para adquirir material para el Laboratorio, mientras solicita que se active la construcción del edificio del Laboratorio en calle Santa Rosa esquina Mendoza.

El laboratorio constaba de un subsuelo de una amplitud tal que comprendía todo lo edificado y se comunicaba con otras dependencias de la Escuela Práctica. (...) En la planta baja existía una amplia sala para demostraciones experimentales, con su mesa-cátedra, con agua, gas, corriente eléctrica de varios voltajes, etc. Esta habitación está ocupada actualmente por la Biblioteca del Instituto. Luego había un largo Salón, muy bien iluminado con ventanales altos, que se destinaba a trabajos prácticos de los alumnos con seis mesas, provistas de todo el material para la experiencia a efectuar; en las paredes, vitrinas con el instrumental depositado. Seguía una pequeña habitación destinada a escritorio y biblioteca; luego el laboratorio de químicabiológica, con mesas especiales para el trabajo químico, campana para gases, vitrinas, estantes para reactivos, etc. Finalmente dependencias sanitarias, depósitos, estabularios, etc. El laboratorio descrito permaneció inalterado hasta el año 1937, en que el Prof. Oscar Orias consiguió su ampliación, aumentando un segundo piso. (...) El Dr. Grandis organizó la enseñanza de la Fisiología Experimental en un grado extraordinario para su época. Todo el sistema de trabajos prácticos, con las mejoras introducidas a partir de 1907 por el Prof. Ducceschi, se mantuvo inalterado hasta 1935, fecha en que el Prof. Orías introdujo otros. (MARSAL, A., 1973, p. 10.) 
Finalmente Grandis, solicitado por su gobierno, parte a Italia en 1906 para asumir el cargo de Profesor Director del Real Instituto de Fisiología de Génova. Cargo que se le planteaba irrechazable, por ser de mayor jerarquía que el que sostenía en la Facultad de Medicina de Córdoba (J efe de trabajos prácticos). Tras darse la baja, el ministro Dr. Joaquín V. Gonzáles, solicita al Prof. Luciani por intermedio del ministro en Roma, que le indique otro profesional. Y allí emerge la figura de Virgilio Ducceschi.

\section{Virgilio Ducceschi, laboratorio y cátedra de Psicología Experimental en Córdoba}

Virgilio Ducceschi, nace el 1 de septiembre de 1871, en Scansano, provincia de Grosseto. Estudió medicina en el Instituto di Studi Superiori in Firenze y luego en 1895 al Instituto de Fisiología de la Universidad de Florencia, dirigido por el Prof. Giulio Fano. De 1900 a 1906 pasó al Instituto de la Universidad de Roma, dirigido en aquel momento por el Prof. Luigi Luciani. Luego de terminar sus estudios de fisiología en Italia, viajó a Estrasburgo para formarse con los profesores de fisiología Golz y J. R. Ewald, y química-biológica con el prof. F. Hofmeister. Terminada sus estudios asume por concurso en 1905, a la titularidad de la cátedra de Fisiología en la Universidad de Palermo, a la que renuncia para viajar a Córdoba.

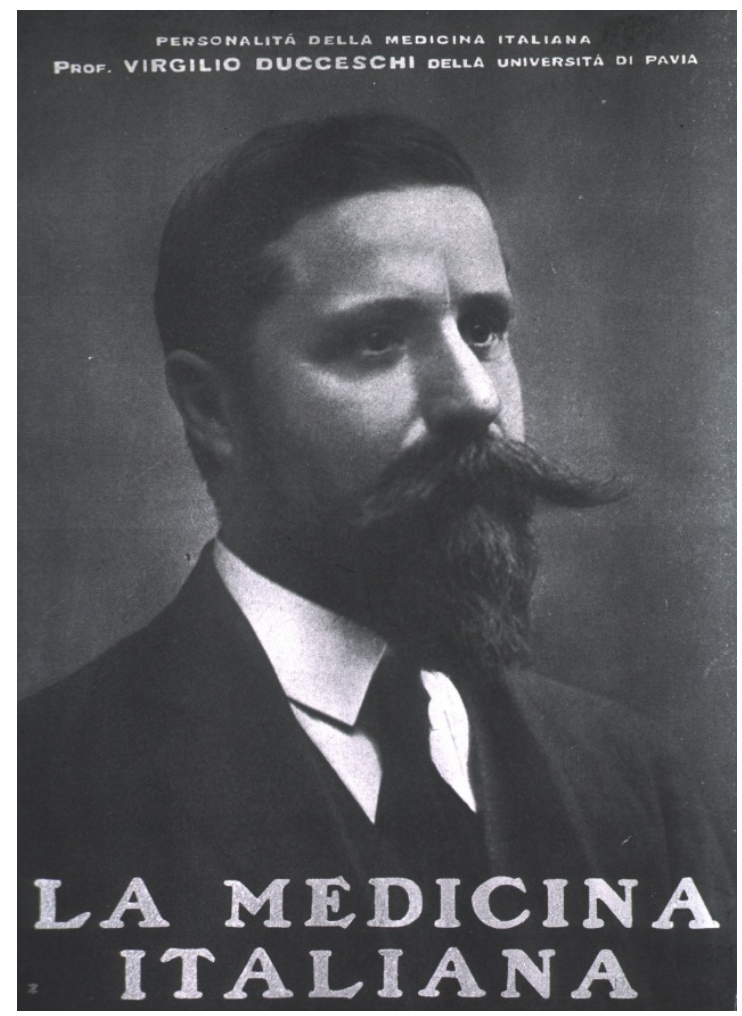


Retrato de Virgilio Ducceschi. U.S. National Library of Medicine, History of Medicine Division. De I mages from the history of medicine (I HM)

Las referencias a la obra del italiano ya estaban presentes en las primeras historias de la Psicología argentina, ya en los clásicos textos de René Gottheld podemos encontrarnos con que:

En la universidad de Córdoba fue profesor de Fisiología y Psicología el Dr. Virgilio Ducceshi, autor de numerosos estudios experimentales e investigador de la técnica psicofísica que describe en su libro Un nuevo registrador mental. El Dr. Ducceschi había sido docente libre de fisiología en la Universidad de Roma. (GOTTHELD, R., 1969b, p. 194.)

Mauricio Papini (1976) documentaba:

Ya en la primera década de este siglo, existían cátedras de Psicología en las principales universidades del país. En Córdoba actuó Virgilio Ducceschi, que era además profesor de fisiología. Sus investigaciones se mantuvieron en el área de la psicofísica (Un nuevo registrador mental) y de la psicofisiología de los órganos sensoriales (Investigaciones anatómicas y fisiológicas sobre aparatos sensitivos del cutis humano; Sensibilidad cutánea y sentido muscular). (PAPINI, M., 1976, p. 322.)

No obstante haber sido referenciado su trabajo por estos pioneros de la historia de la psicología, los estudios posteriores parecen haber perdido la pista de los desarrollos del italiano en Córdoba. El único trabajo que ha sido realizado hasta el momento sobre las experiencias de Ducceschi es el de Marsal (1973), al que hemos accedido tras una intensa búsqueda en los archivos de la Biblioteca Mayor de la ciudad de Córdoba, ya que no fueron clasificados en las nuevas bases de datos actuales. De allí la dificultad de dar con este material, así como el de Ducceschi.

El trabajo de Marsal (1973) sobre Ducceschi es esclarecedor y eleva al Laboratorio de Fisiología y Psicología Experimental al más alto nivel:

Ducceschi llegó a Córdoba y se hizo cargo del Laboratorio, montado con una riqueza de medios de trabajo, que pocos en el mundo podrían presentar en aquella época. Los instrumentos que había comprado su antecesor el Prof. Grandis lo fueron en tal cantidad, que veinte años después aún alcanzaban para las necesidades corrientes y el inventario de tales compras demuestra su abundancia. Ducceschi fue un trabajador incansable y no se preocupó de otras actividades, de tal manera que su olvido total de la política universitaria sirvió como precedente, años después, para rechazar la pretensión de otro profesor en parecidas condiciones (Arch. Univ.), que deseaba formar parte del 
claustro universitario, en los actos electorales y el gobierno de la casa.

El Prof. Ducceschi tenía especial interés en la fisiología de los sentidos y el sistema nervioso. Propuso la creación de una Cátedra de Psicología Experimental, que resultaba algo extraordinariamente nuevo, al comienzo del siglo. La Universidad y el Poder Ejecutivo de la Nación crearon esa Cátedra, pero aparecieron dificultades al querer fijar su sede en la Facultad de Derecho y Ciencias Sociales, cuyo Consejo Directivo rechazó la idea, argumentando que debería originarse en la propia Facultad. El 2 de mayo de 1907, al tener noticias que se dispondría de la correspondiente partida financiera, formó una terna compuesta por el Dr. Benjamín Otero Capdevila, Dr. Exequiel Morcillo y Dr. Eleuterio Ríos.

El Poder Ejecutivo Nacional dictó un decreto el 15 de junio de 1907 (Arch. Univ.) Firmado por el Presidente Dr. José Figueroa Alcorta y el ministro Dr. Federico Pinedo, haciendo amplias consideraciones sobre la oportunidad de crear en Córdoba 11 cátedras libres, en aquellas materias de importancia excepcional y que no correspondían a ninguna Facultad en particular, sino a todas las que pudieran estar interesadas. En los fundamentos del decreto se hacían consideraciones muy elevadas e interesantes sobre este tipo de cátedras y se lamenta que el estatuto de la Universidad de Córdoba no hubiese creado esta enseñanza, permitiendo la incorporación de personas calificadas. En el articulado, se crean las cátedras libres de Psicología Experimental y la de Pedagogía (que funcionaría, esta última, en el Colegio de Monserrat). Se designó profesor titular en Psicología al Dr. Ducceschi, cuyas retribuciones por contrato se aumentaban con lo correspondiente a la nueva función. Finalmente se invitó a la Universidad de Córdoba a revisar sus estatutos, si lo creía conveniente.

El Dr. Ducceschi se mantuvo en la Cátedra de Psicología Experimental desde el 15 de junio de 1907 al 28 de febrero de 1919 Arch. Univ.). Organizó la enseñanza y la investigación con carácter vocacional. La clase inaugural del año 1912 fue publicada en la Revista del Círculo Médico de Córdoba Año III (febrero de 1913), N. ${ }^{\circ} 2$, y versó sobre "La criminología moderna", en la que rindió tributo a la escuela argentina. (...) El autor de esta biografía recuerda haber visto en 1922-24, una Sección del Laboratorio de Fisiología Humana denominada Psicología Experimental; desafortunadamente en aquellos años el maestro ya no se encontraba en Córdoba.

El prestigio que tomó el Laboratorio de Córdoba llevó a la Facultad de Medicina de Buenos Aires a ofrecer al Prof. Ducceschi su Cátedra de Fisiología, que luego ocuparía, con el más grande de los éxitos, el Dr. Bernardo A. Houssay. Ducceschi no aceptó el ofrecimiento... (MARSAL, 1973, p. 1718. No hemos podido encontrar el archivo al que alude Marsal.) (Resaltado del autor de este artículo). 
Como mencionamos, la enseñanza de la Psicología en Argentina, había sido inaugurada por Horacio Piñero (1896) y, en 1906, un segundo curso de psicología. En Buenos Aires el positivismo permitió la convivencia de las prácticas experimentales con la reflexión filosófica, de allí que los primeros cursos estuviesen vinculados con la Facultad de Filosofía. En Córdoba, la enseñanza que Ducceschi desarrolla de la Psicología (1907), según Marsal, se distancia de los planteos filosóficos y se instala definitivamente en la Facultad de Medicina. A diferencia de lo ocurrido en Buenos Aires, el discurso filosófico estaba dominado por el pensamiento religioso. De modo que el desarrollo de la Psicología como ciencia se centró en el ámbito médico. Algunos rastros de esa experiencia fueron documentados por uno de los primeros historiadores de la Psicología argentina y uno de sus más destacados intelectuales, José Ingenieros:

Recordemos que la enseñanza superior de la Psicología se imparte actualmente en las universidades de Córdoba, La Plata y Santa Fe, según los criterios y métodos que la han elevado a la dignidad de ciencia. En Córdoba es profesor de Fisiología y Psicología el doctor Virgilio Ducceschi; en la Plata enseñan Carlos Rodríguez Etchart, Psicología general, y Rodolfo Senet, psicología pedagógica y anormal. En Santa Fe el doctor José Oliva, que comenzó sus cursos explicando psicología neotomista y enseña actualmente psicología biológica. (INGENIEROS, J., 1910, en VEZZETTI 1996, p. 70.) (Resaltado del autor de este artículo).

\section{Las producciones escritas de Ducceschi}

Si bien nos hemos centrado en los aspectos socioinstitucionales y biográficos de la experiencia investigativa e intelectual de Ducceschi en Córdoba, es importante mostrar algunos de sus trabajos escritos. Pero debemos consignar que resta un análisis discursivo de mayor profundidad proyectado en futuras investigaciones.

Sus trabajos de psicofisiología estarían ubicados dentro de un selecto grupo de investigadores argentinos e italianos:

Entre los fisiólogos de principios de siglo son de interés los estudios sobre psicofisiología realizados por Valentín de Grandis, Mariano Alurralde, Pastor Anargyros, Andrés F. Llobet, Franck L. Soler y los ya citados de Horacio Piñero y Virgilio Ducceschi. Con los trabajos de biología y neurobiología de Christofredo Jakob y sus discípulos, alcanzaron gran importancia los estudios filológicos del sistema nervioso central y particularmente del cerebro. (GOTTHELD, 1969b, p. 197.) 
Para poder mostrar algunas de sus líneas de investigación, realizaremos una selección (un tanto arbitraria) de la bibliografía que hemos relevado. Queda pendiente para investigaciones posteriores un análisis profundo y sistemático de toda su obra escrita. Dentro de la bibliografía que hemos localizado, podemos dividir, heurísticamente, su producción en tres momentos: Sus escritos previos a su estancia en Córdoba (1903-1906), los que desarrolla en la ciudad mediterránea (1907-1918) y los que produce en Italia (1919-1925).

En los trabajos del primer período se pueden notar las influencias de su formación con Giulio Fano y Luigi Luciani. Como mencionamos también realizó un viaje de especialización a Estrasburgo en donde se formaría con los profesores de Fisiología Golz y J. R. Ewald, y química-biológica con el Prof. F. Hofmeister. Eminentemente en fisiología, algunos de sus estudios versaron sobre: Di una modificazione macroscopica del sangue. Che precede la coagulazione (DUCCHESCHI, 1903a.); Un nuovo metodo di sfigmografía (DUCCESCHI, 1903b.) y Sulla fisiología Della respirazione. Osservazioni su di un caso di fistola bronchiale nell'uomo (DUCCHESCHI, 1906.)

Los trabajos que hemos podido relevar en el período en que se desempeñó en Córdoba sostienen esa fuerte influencia en fisiología y psicofisiología. Gran parte de sus aportes fueron publicados en dos tomos titulados Trabajos del laboratorio de fisiología (DUCCESCHI, V., 1908, 1915.). Dentro del primer tomo se encuentran trabajos de fisiología de gran importancia como el experimento que realizó sobre la leche de los marsupiales en donde hay una referencia a las famosas investigaciones del nervio gástrico de Pavlov (DUCCESCHI, V., 1908, p. 9.). Los trabajos sobre aprendizaje del fisiólogo ruso serán referenciados recién con la presencia del alemán Nicolai Friedrich, quien será el sucesor de Ducceschi en la cátedra de Fisiología Experimental. En 1912 publica un tomo con una completa compilación de resúmenes de su contribución científica, titulado Contributi Scientifici (DUCCESCHI, V., 1912). De relevancia para nuestro tema, encontramos Sobre una alteración de la sangre producida por la intoxicación alcohólica (DUCCESCHI, 1914a.) y La colesterinemia en la intoxicación alcohólica. Córdoba (DUCCESCHI, 1914b.) en donde estudia las modificaciones químicas que la intoxicación por alcohol etílico ocasiona en los líquidos y tejidos del organismo, particularmente el intercambio de la colesterina, y un trabajo junto al Dr. Barilari, quien fue agregado al Laboratorio de Fisiología durante 1914 y 1917, jefe técnico hasta 1920: Sobre la patogénesis de la intoxicación alcohólica (DUCCESCHI, V. y BARILARI, D., 1915.). En 1913 publica en la Revista del Círculo Médico de Córdoba la clase inaugural de un curso de antropología criminal y psicopatología médico-legal dictado en la Facultad de Derecho y Ciencia Sociales que se publicaría con el título de La 
criminología moderna (DUCCESCHI, V. 1913.). Éste texto es uno de los pocos que hemos hallado en el cual se presenta abiertamente una conjunción de líneas de investigación propias del positivismo. En donde vemos emerger una concepción positiva de la psicología. De modo que es en estas épocas que podemos ver aparecer la articulación que la Psicología científica realiza a partir de dos modelos: la Fisiología y la psicopatología (TALAK A., 2000a). El texto que allí expone tiene por objeto desarrollar las principales problemáticas de la criminología, sin intención de presentar una "ciencia nueva", y reconoce allí a varios impulsores argentinos ya reconocidos: José Matienzo, Luis M. Drago, Antonio Dellepiane, Cornelio Moyano Gacitúa, J osé Ingenieros, Osvaldo y Norberto Piñero, Rodolfo Rivarola, Francisco de Veyga. (DUCCESCHI, V., 1913, p. 57.) Dispone también una crónica de los desarrollos científicos en el área, comentando el crecimiento de la criminología en tres áreas de estudios, la Antropología, la Psicología y la Sociología Criminal. Áreas que delimitará mencionando a sus principales representantes. El espacio de la psicopatología emerge principalmente ligado a la Criminología y a la medicina:

(...) la nueva criminología tuvo numerosos cultores y principalmente sus iniciadores entre psicólogos, fisiólogos y psiquiatras. Por este motivo ocupa hoy un médico una cátedra de la facultad de Derecho. (DUCCESCHI, V., 1913, p. 59.)

Fundamentalmente se puede ver que la incipiente psicología estuvo particularmente vinculada a la criminología, no tanto a la clínica ni a la educación, aunque algunas incursiones se realizaron a fines de la década del veinte y el treinta. En este texto, no pierde ocasión para mencionar al hito que representó la creación del Laboratorio de Wundt y expone que la fisiología y la biología tienen una íntima relación con la antropología y Psicología médico-legal.

El positivismo, que hace una tardía aparición en Córdoba, desarrolló singularidades que no responden a las mismas condiciones que en Buenos Aires. El particular ámbito cultural de la Córdoba clerical atacó sistemáticamente a los avances de la ciencia. La oposición a la Psicología positiva verá su máxima expresión en el "Compendio de Psicología contemporánea" que el Padre Liqueno escribiese en 1919 (LIQUENO, 1919), sobre el que no disponemos espacio en este escrito para desarrollar. Baste mencionar su compromiso intransigente con la Psicología neotomista. Un recorte del periódico conservador, Los Principios, demuestra la tensión que las conferencias de Psicología Experimental de Ducceschi producían en el medio Cordobés: 


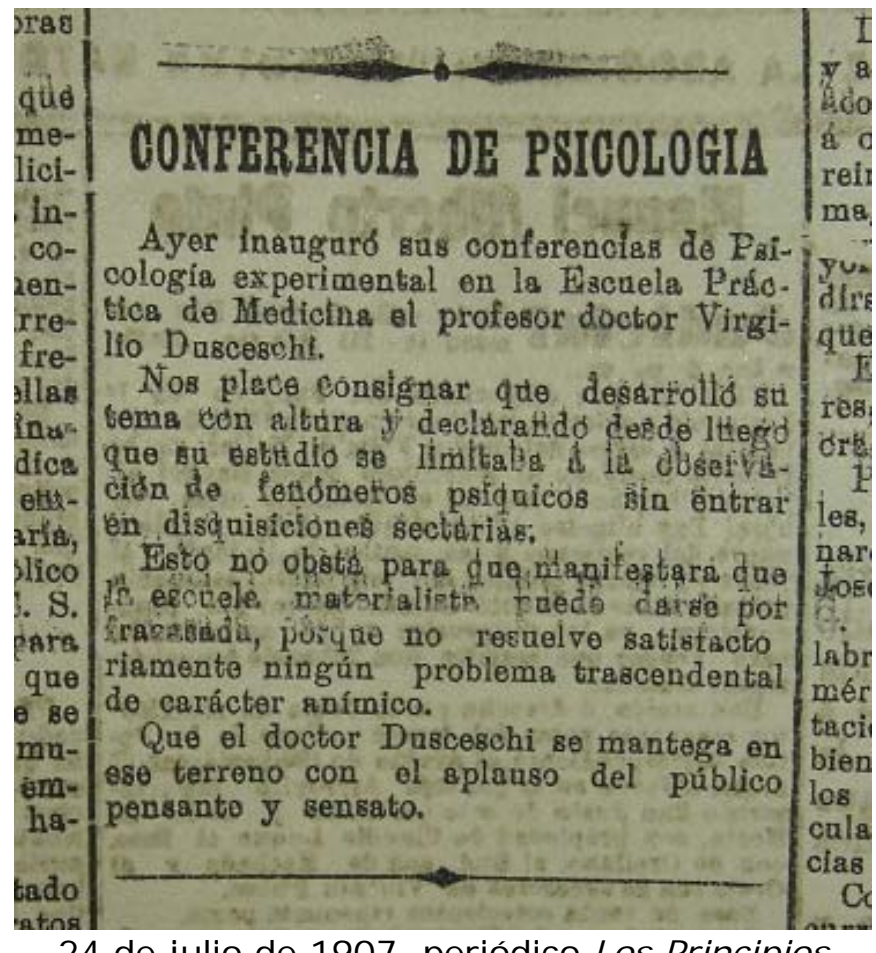

24 de julio de 1907, periódico Los Principios

Sobre sus experiencias en el laboratorio de Psicología Experimental hemos encontrado pocas referencias, pero según Duccheschi, él realizó estudios sobre:

Las modificaciones aportadas por algunos estados somáticos sobre el desenvolvimiento de las actividades psíquicas elementales y para determinar bajo cual aspecto se manifiestan en las varias edades algunas formas fundamentales del trabajo mental. (DUCCESCHI, V., 1910, p. 8.)

Dentro del tomo que reúne los trabajos del laboratorio de fisiología, en su primera serie (1907-1908) encontramos investigaciones realizadas sobre "el mal de montaña" o "puna" en Sudamérica (DUCCESCHI, V., 1908.).

También colaboraría con Ducceschi, Juan F. Cafferata, quien había trabajado como adscripto en el Laboratorio de Wernicke, de 1897 a 1899. Publicaría en la revista de la Universidad un trabajo realizado en el Laboratorio de Fisiología Experimental: El coeficiente tóxico de las bebidas alcohólicas de uso común (CAFFERATA, 1919.).

Seguidamente nos dedicaremos a tratar un artículo particularmente interesante que fue publicado dentro de la serie de trabajos realizados en su estancia en Córdoba: Un registrador mental (DUCCESCHI, 1910a.).

Finalmente, dentro de las publicaciones del período en que retoma sus investigaciones en Italia, podemos encontrar producciones que continúan la línea planteada en Córdoba: Una dimostrazione prática 
sui muscoli Della laringe (1923), Sul mecanismo Della hipoglicemia da insulina (1924); Azione del simpatico cervicale sui la tonicitá del muscoli dell'oreccio esterno (1925); Dulla tonicitá dei muscoli intrinseci Della laringe (1925).

Todos estos trabajos muestran una orientación psicofisiológica y fundamentalmente fisiológica de sus investigaciones. Si bien no hemos encontrado todavía mayores informaciones sobre los contenidos del curso libre de psicología, podemos conjeturar que se orientaban mayormente a tratar las temáticas que hemos documentado aquí. No pretendemos hacer aquí un análisis exhaustivo de su obra, tarea que queda pendiente para futuras investigaciones, sino que buscamos mostrar documentación y bibliografía que contribuye a pensar en la obra de Ducceschi como un enclave cultural, que se distancia de los desarrollos realizados en Buenos Aires.

\section{Referencias a la Psicología Experimental, el registrador mental de Virgilio Ducceschi}

A pesar de haber incursionado en la Psicología Experimental y su enseñanza, no hemos podido encontrar mayores producciones escritas sobre el tema. Además, pudimos constatar que Ducceschi no aparece dentro de los inscriptos en lo que fue, en 1908, la primera Sociedad Argentina de Psicología (KLAPPENBACH, H., 1997, p. 179.). El caso de Virgilio Ducceschi, tal como lo menciona Buch (en MONTSERRAT, M.; ANDERMANN, J. 2000), implicó un "enclave" que no solo desarrolló la Psicofisiología, sino que innovó en el ámbito experimental, aún en un medio tenazmente tradicionalista. En Córdoba la Psicología Experimental encuentra una expresión original, que implica un esfuerzo por la innovación. En 1910, Virgilio Ducceschi publica Un registrador mental (comunicación y aparato presentados al Congreso Internacional reunido en Buenos Aires en 1910). El mismo trabajo sería presentado en una comunicación en el Congreso Internacional de Psicología en Genova (1909) (MACEDA, G., 1927, p. 81.). También presentaría Una ley del movimiento animal al mismo Congreso internacional de Buenos Aires (1910).

Un párrafo que escribiese Américo Foradori en su texto La Psicología en la República Argentina es retomado por Gottheld y reproducido por Papini (1978). Es muy interesante la síntesis en la que se ubica Ducceschi con su ponencia del registrador mental:

Los trabajos, tomados en su conjunto, indican la tendencia a buscar la explicación en la Fisiología y la inmediata aplicación en la Pedagogía, y pusieron en evidencia una fecunda vida de laboratorio, ofreciendo importantes investigaciones 
realizadas en Buenos Aires, La Plata, Córdoba y Santiago de Chile. La Psicología fue presentada en el Congreso en aspectos científicos diversos: 1) anátomo fisiológico: por Jakob, Roveda, Balaro y Borda; 2) experimental y de investigación, para el conocimiento del sujeto aislado o en grupos con sus aplicaciones correspondientes, por Mann, Anagyros, Ducceschi, Mercante, Senet, Quadri, Moreno, Scultze, etc.; 3) anormal y patológica, con aplicaciones a la Pedagogía, por Piñero, Senet, Vidal, Cometto, Valdez, y otros; 4) subjetivo y doctrinario, por Rodriguez Etchart, Mouchet, Pascarella, etc.; 5) social y religioso: por Vergara, Sisson y Jara; 6) pedagógico, por Keiper, Romero, Brest, Morel; 7) criminológico, por Silva Cruz y Ramirez; y 8) comparada, por Onelli (PAPINI, 1978, p. 333.).

Los tiempos de reacción, tan estudiados, conllevaban algunas dificultades, en particular la cronometría de las reacciones. Asociados a los estudios astronómicos y a las telecomunicaciones, las maquinarias de registro de reacción fueron complejizándose. Scherman desarrolla en varios puntos de su tesis (SCHERMAN, 2009, p. 70.) la importancia de la medición del tiempo. El problema de consolidar una medición temporal unificada, estaba en la base del sistema económico y social de fines de siglo XIX. Pues debían compartir un mismo sistema horario diversas áreas y actividades, que sin embargo, resultaba difícil de coordinar por la incipiente tecnología utilizada. Los laboratorios de Psicología tomarían el problema de la medición de los registros de la astronomía, pues comenzó a notarse la dificultad del registro de un mismo fenómeno en manos de diversos observadores. Esta actividad arrojaba diferencias entre los registros, lo que lanzó a la carrera por el estudio de esas diferencias. Scherman (SCHERMAN, 2009, p. 71 citando a PAOLANTONIO, S., y MINITTI, 2004.) destaca que:

\begin{abstract}
Argentina fue un país adelantado para su época en ese sentido, el Observatorio Astronómico Nacional (ONA) fue creado en 1871 y cumplió, entre otras, esa misma función de coordinación entre las horas locales, contribuyendo a coordinarlas y unificarlas. A fines del siglo XIX, cuando en los ámbitos universitarios o intelectuales europeos se hablaba de Argentina, la referencia era Córdoba, como expresión de los más avanzados en ciencias en Latinoamérica.
\end{abstract}

Los instrumentales para el registro de reacciones temporales eran sumamente complicados e imponían constantemente dificultades técnicas que alteraban los resultados de la experimentación. Desde el cronoscopio de Hipp, realizado por Mathias Hipp, relojero y comerciante alemán, había sido basado en el instrumental utilizado en la guerra que medía la velocidad de los proyectiles. El uso de este dispositivo, sumado a la emergencia de la teoría de la apercepción, permitió un cambio en la consideración de los procesos psicológicos. 
El problema a resolver experimentalmente era el de poder medir los tiempos muy breves con toda exactitud, que era el que una representación simple permanecía en la conciencia, esto se lograba determinando el tiempo entre representaciones generados por la percepción a estímulo sensoriales acústicos o visuales (SCHERMAN, 2009, p. 75.).

El problema de la precisión cronométrica de los eventos era de suma importancia en la experimentación; es por ello destacable el intento de Ducceschi de realizar un aporte a la Psicología Experimental, brindando un nuevo aparato, más sencillo, a su criterio. Veamos de qué se trató el invento de Ducceschi:

Por cuanto los métodos directos sean preferibles en el estudio de las leyes del trabajo mental, presentan, en las formas hasta ahora propuestas, inconvenientes no leves y especialmente el de obtener solo el resultado conjunto del trabajo ejecutado en un intervalo más o menos largo de tiempo (no menor de cinco minutos y en algunos casos quince minutos); en este caso, si pueden apreciarse las oscilaciones en cantidad del trabajo mental, nos faltan los datos más minuciosos y precisos relativos al modo de desarrollarse, es decir, a sus caracteres cualitativos. Otro inconveniente es el de tener que escribir el sujeto mismo largos dictados, o los resultados de cálculos o de otras operaciones mentales. Estas consideraciones me indujeron a buscar un procedimiento que permitiera analizar por medio de la registración gráfica, en su sucesión y en todos sus particulares, en forma fiel y al mismo tiempo tan simple que permitiera numerosas investigaciones, la forma en que se desarrolla una determinada calidad de actividad psíquica, siguiendo al mismo tiempo un método de valuación directa. Hice construir para este fin un aparato que se basa en el siguiente principio general: registrar gráficamente la sucesión con la cual se cumple una serie continua de operaciones mentales breves, relativamente simples y fáciles, y poco desiguales entre sí, pero que ocupan en modo completo y permanente la atención del sujeto durante todo el tiempo del experimento. El aparato que describiré responde a este principio, y registra gráficamente el término de cada cálculo, indicando al mismo tiempo, donde se necesite, si la operación mental ha sido exacta; por eso propongo el nombre de Registrador mental. La curva que se obtiene expresa fielmente el ritmo de la actividad mental, correspondiendo a la forma estudiada, con todas las variaciones y oscilaciones, indica la cantidad relativa del trabajo mental en la unidad de tiempo, y contiene también algunos datos cualitativos (faltas u otras particularidades) que se refieren a este (DUCCESCHI, 1910a). 


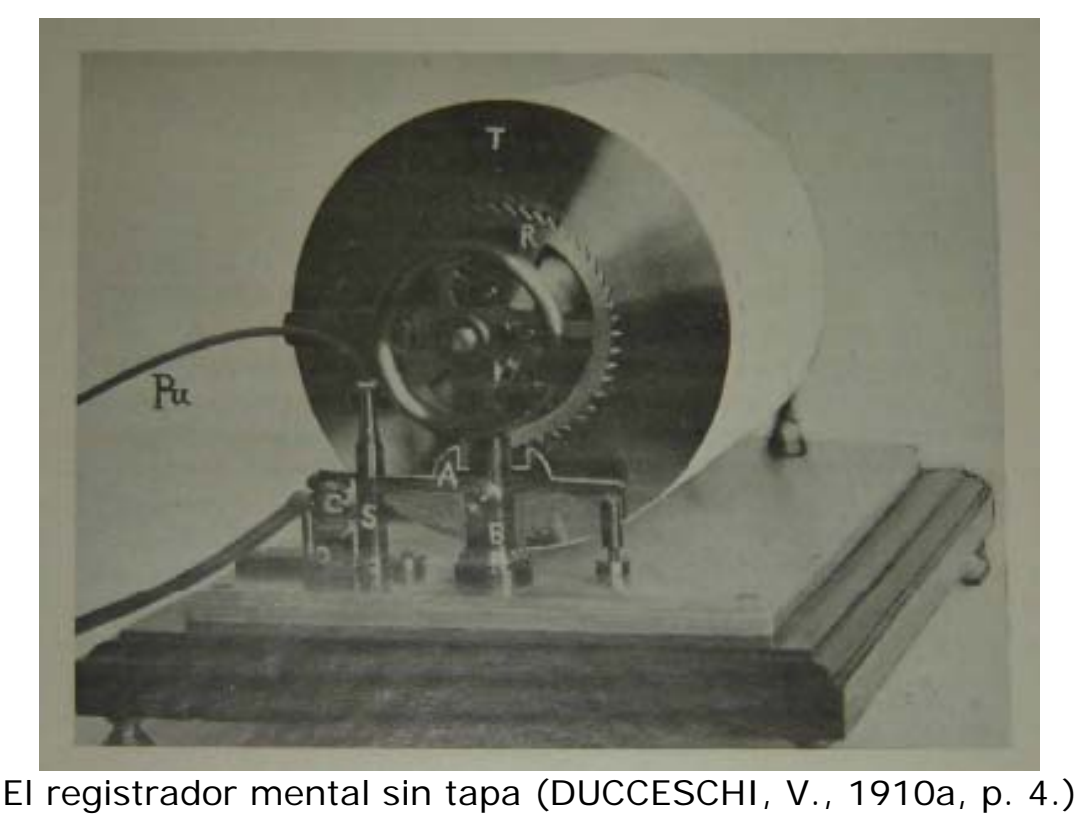

\section{Continúa Ducceschi:}

Para las investigaciones a cuyo efecto se ha construido el instrumento descripto, se necesita una serie de pruebas (tests) mentales, cuyo contenido varía según los casos. Se tratará de números si se emplea el método de los cálculos y se estudia el trabajo mental y la atención; de palabras, si se estudia los fenómenos pertenecientes especialmente a la asociación de las ideas o de la imaginación. Cuando se trata de cálculos mentales es necesario preparar una tabla o clave de ellos conteniendo las operaciones resueltas, en el mismo orden en que se encuentran en la prueba sometida al sujeto y que sirven al contralor del experimento para apuntar los errores. (...)

Con este procedimiento se evita, además, una de las más graves causas de complicación existente en muchos métodos psicológicos, que previene de la necesidad que el sujeto escriba él mismo los resultados de las operaciones mentales, agregándose así al trabajo intelectual un trabajo manual que estorba -en medida y en forma muy diferente, y difícilmente valuable- los varios sujetos de experimento (DUCCESCHI, 1910a, p. 5.).

El aparato representa con trazos diferentes datos:

El examen de los trazados obtenidos dará los siguientes datos principales:

a) duración del experimento;

b) número total de las operaciones mentales ejecutadas;

c) número de las operaciones mentales en la unidad de tiempo elegida como medida;

d) ritmo con el cual se siguen las operaciones mentales;

e) influencia de las condiciones psíquicas temporáneas (modificaciones durante el experimento, variaciones horarias, 
diarias, etc.) y permanentes, sobre la curva de las operaciones;

f) influencia de circunstancias externas;

g) número conjunto de los errores en la unidad de tiempo, en varios pequeños intervalos de la curva o en diversas condiciones experimentales.( DUCCESCHI, 1910a, p. 7.)

El aparato además, se adapta a diferentes tipos de tests, según la investigación que se quisiera realizar.

El invento fue valorado por importantes figuras de la Psicología del momento, por ejemplo en La psicología en la república Argentina, Ingenieros (1909) dirá:

El profesor Virgilio Ducceschi, de la Universidad de Córdoba, es autor de numerosos estudios experimentales, entre los que nos interesa singularmente la descripción de "Un nuevo registrador mental". (INGENIEROS, 1909, en VEZZETTI, 1996, p. 57.)

Florencia Ibarra comenta que por esos años se utilizó un registrador mental similar al de Ducceschi, modelo Mo-Alberti, en el Laboratorio de Psicofisiología de El Palomar. Utilizado para indagar sobre la actividad psicomotriz (tiempos de reacción), atención y memoria (concentración y fluctuación de la atención; extensión de la atención; rapidez de la percepción; memoria y reacciones psicomotrices) (ROSSI, IBARRA, KIRSCH, 2010.). Sin embargo, Ducceschi parece atribuirse la autoría del aparato, pues no señala en su artículo ninguna referencia respecto de su origen. Otros autores plantean que, efectivamente, Ducceschi inventó el aparato "Fue creador también de un aparato para registrar el tiempo de operaciones mentales sencillas" (BUCH A. en MONTSERRAT, M.; ANDERMANN, J. 2000,31 ). Queda pendiente un análisis comparativo del aparato con otros similares para determinar la originalidad del invento.

\section{Conclusiones}

Hemos podido documentar las condiciones socioinstitucionales de la existencia de un laboratorio de Psicología Experimental en Córdoba (1907) y la cátedra libre de Psicología Experimental, ligados a la Facultad de Medicina. Las mencionadas condiciones implicaron la radicación del laboratorio y la cátedra en el ámbito médico, lo cual lo diferencia de las cátedras de Psicología de Buenos Aires, que se instalan en la Facultad de Filosofía. La selección que realizamos de sus publicaciones (que de ningún modo busca ser exhaustiva) muestra que sus aportes fueron realizados dentro de los campos de la fisiología, Psicofisiología y Psicología Experimental. Estos, sin haber sido analizados en profundidad contribuyen a la hipótesis de que sus 
aportes a la ciencia no se adaptaron al mismo modelo docente e investigativo que predominó en Buenos Aires. Habiéndose formado estrictamente en fisiología, tanto en Italia como en Alemania, parece haber adoptado un modelo de investigación no ligado exclusivamente a la docencia, ni a planteos filosóficos como los que se presentan en los cursos de la ciudad del puerto. La calidad de las experiencias que realizase Ducceschi es indudable, pues fue preconizada por los más importantes médicos dedicados al estudio de la temprana Psicología en Argentina, por caso, J osé Ingenieros. Teniendo en cuenta además, que el espacio de investigación fue preparado por el Dr. De Grandis, quien fuera maestro del grupo de investigadores que desarrolla una gran tarea en Buenos Aires, en torno de la figura de Horacio Piñero. Piñero y Ducceschi vivieron en una época paralela, trabajando en un espacio epistémico similar. Pero tal como menciona Buch:

(...) el escepticismo y la prudencia expresados por Ducceschi, su mesura intelectual y personal, se contraponía de manera notable con la personalidad de Piñero. Con formaciones radicalmente distintas, la profesionalidad del científico cauto y dedicado a su disciplina contrastó en sus escasos resultados sociales con el entusiasmo desbordante del fisiólogo y psicólogo amateur. Lo único que diferenciaba de manera significativa sus contextos era la distancia cultural existente por entonces entre la cosmopolita y agitada vida de la ciudad de Buenos Aires frente a la dormida y clerical ciudad de Córdoba.

De tal modo, a lo largo de más de una década en las Facultades de Medicina de Córdoba y de Buenos Aires se desarrollaron dos programas de actividad distintos. En

Córdoba se realizaron numerosas investigaciones originales de incuestionable calidad, vinculando a Ducceschi a las redes disciplinarias de la fisiología italiana. Se trató en cierto modo de un "enclave" de la fisiología italiana en la Argentina. En Buenos Aires se estableció, por el contrario, un programa de reproducción experimental dirigido por un médico y fisiólogo ocupado en múltiples tareas. La historia de los vencedores, el relato del éxito, pasa por Buenos Aires. (BUCH A., en MONTSERRAT, M.; ANDERMANN, J. 2000, pp. 28-29.) (Resaltado del autor de este artículo).

La última reflexión de Buch, no solo alude al centralismo que ha marcado la historia y la actualidad de la política y las ciencias argentinas, sino también a una profunda divergencia en torno a la formación que separa a las publicaciones de Piñero y Duccheschi. Lo cual contribuye a abonar a la hipótesis que indica que las prácticas y discursos que se despliegan en Buenos Aires difieren de las realizadas en Córdoba. La combinación del mencionado centralismo con la innegable existencia de una peculiaridad cultural cordobesa ligada al conservadurismo clerical, puede explicar la poca visibilidad de las 
producciones del italiano en Córdoba. No por ello han tenido menos rigor que los estudios en Buenos Aires. Resta la realización de una investigación profunda sobre su obra escrita y una reconstrucción biográfica y socio-institucional de su formación, de su estancia en Córdoba y su posterior desempeño en Italia. Hasta ahora, nuestra primera aproximación ha mostrado la existencia de prácticas en la Psicología Experimental y la docencia universitaria de la Psicología como ciencia en Córdoba a partir de la presencia de Ducceschi (1907). Confiamos en que esta nueva línea de investigación pueda abrir nuevos caminos que sitúen a los tempranos desarrollos cordobeses en la historia de la Psicología argentina y latinoamericana. En esta línea, esperamos continuar trabajando con los médicos, alienistas y juristas que se dedicaron al problema de la criminalidad y su evaluación psicológica.

\section{Referencias}

ARDILA, R. Desarrollo de la psicología latinoamericana. Revista Latinoamericana de Psicología. Fundación universitaria Konrad Lorenz, Bogotá, Colombia. 1969 Vol. I, no 001, pp. 63-71 Disponible en: $\quad$ <http://redalyc.uaemex.mx/redalyc/pdf/805/80510106.pdf> Accesado en_8 de feb. 2012.

ARDILA, R. La psicología en América latina. Pasado, presente y futuro. México: Siglo XXI. 1986.

- La revista latinoamericana de psicología: Los primeros 30

años. Revista latinoamericana de Psicología. Fundación universitaria Konrad Lorenz, v. 30, n. 3, p. 393-400 1998, Disponible en: $\quad<$ http://redalyc.uaemex.mx/redalyc/pdf/805/80530301.pdf> Accesado en 8 de feb. 2012.

BUCH A. El papel de los fisiólogos extranjeros en la Argentina de principios de siglo o acerca de la "nacionalidad" del mate amargo. En MONTSERRAT, M.; ANDERMANN, J.. La ciencia en la Argentina entre siglos: textos, contextos e instituciones. Buenos Aires. Ed. Manantial, p.19-33, 2000.

CAFFERATA, J. F. Investigaciones sobre el coeficiente tóxico de algunas bebidas alcohólicas de consumo habitual en el país. Revista de la Universidad de Córdoba, Córdoba, v.6 , n.1, p. 58-67, 1919. Contratación de Virgilio Ducceschi. 1907- Contratación del Dr. De Grandis. 1904. Libro de Anotaciones de Secretaría Memorándum (1910-1940). Archivo de la Facultad de Ciencias Médicas Universidad Nacional de Córdoba.

DUCCESCHI, V. Di una modificazione macroscopica del sangue. Che precede la coagulazione. Rendiconti Della R. Accademia dei Lincei. Classe di scienze fisiche, matematiche e naturali.

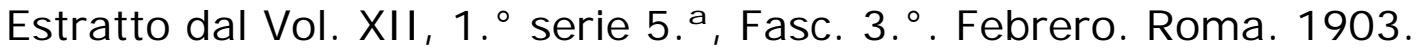


. Un nuovo metodo di sfigmografía. Estratto dell'archivio di

Fisiología. Vol, I Fasc. I. Noviembre. DAll'Institutto di fisioloogía Della universitá di Roma, Diretto Dai Prof. L. Lucciani. 1903.

. Sulla fisiología Della respirazione. Osservazioni su di un caso

di fistola bronchiale nell'uomo. Renconti Della R. Accademia dei lincei. Classe di scienze fisiche, matematiche e naturall. Estratto del Vol. XV. $1 .^{\circ}$ Sem., serie 5. a , Fasc. $4 .^{\circ}$ - Seduta del 18 de febbraio. Roma. 1906.

Trabajos del laboratorio de fisiología. Serie 1.a (19071908). Córdoba: La Italia, Biffgnand, 1908.

. Un registrador mental. Congreso internacional. Buenos Aires. 1910a

. Trabajos del Laboratorio de Fisiología, serie $2^{a}$ (19091910); Córdoba, Talleres "La I talia" de A. Biffgnand, 1910b;

. Contributi Scientifici. Córdoba. La Italia, 1912.

. La criminología Moderna. Lección inaugural del curso libre de antropología criminal y psicología médico-legal dictado en la Facultad de Derecho y Ciencias Sociales de la Universidad de Córdoba en el año escolar de 1912. Revista del Círculo Médico de Córdoba. Año III, n. 2. Febrero, p. 57-76, 1913.

Sobre una alteración de la sangre producida por la intoxicación alcohólica. Revista Médica, Córdoba, n. 3 y 4, 1914a.

La colesterinemia en la intoxicación alcohólica. Córdoba. Laboratorio de Fisiología de la Facultad de Ciencias Médicas Universidad Nacional de Córdoba. Bs. As. Las Ciencias. Buffarini. $1914 b$

. Trabajos del laboratorio de fisiología. Serie 3. a (19111914). Córdoba: Cubas. 1915.

Una dimostrazione prática sui muscoli Della laringe. Estratto dell'Archivio di fisiología, vol XX, Fasc. VI. Firenze. 1923.

Sul mecanismo Della hipoglicemia da insulina. Dal Bollettino Della Societá Medico-Chirurgica di Pavia. Anno XXXVI - Fasc. 4 - 1924. Instituto di fisiología Della Universita di Pavia. 1924.

Insuline et glycolyse. Estratto delle archivies I taliennes de Biologie, Fasc. II. Vol LXXLV. 1926.

Azione del simpatico cervicale sui la tonicitá del muscoli dell'oreccio esterno. Estratto dall'Archivio di fisiología. Vol XVII. Fasc. I-II. Novembre 1919 Gennaio 1920. Instituto Fisiológico Della Universita di Pavia. 1920.

. Il compito Della fisiología sociale. Estratto dall' Archivio di físiologia, volume XXIII - Fascicolo 1, Firenze, 1925.

. Dulla tonicitá dei muscoli intrinseci Della laringe" Estratto dall archivio di fisiología. Volume XXIII- Fasc. I. Firenze. Tipografia Luigi Niccolai. 1925. 
DUCCESCHI V.; BARILARI D. Sobre la patogénesis de la intoxicación alcohólica. Segunda nota. Laboratorio de Fisiología de la Facultad de Ciencias Médicas Universidad Nacional de Córdoba. Bs. As. Las Ciencias. Buffarini. 1915.

GARZÓN M., F. Historia de la Facultad de ciencias médicas. Tomo III. Córdoba. Imprenta de la Universidad. 1927.

GOTTHELD, R. Historia de la psicología en la Argentina. (Primera Parte) Revista Latinoamericana de Psicología, Bogotá, v. 1, n. 001, p. 13-33, 1969a. Disponible en: <http://redalyc.uaemex.mx/redalyc/pdf/805/80510103.pdf> Accesado en: 8 feb. 2012.

GOTTHELD, R. Historia de la psicología en la Argentina. (Segunda parte) Revista Latinoamericana de Psicología, Bogotá, v. 1, n. 2 y 3, p. 183-198. 1969b. Disponible en: <http://redalyc.uaemex.mx/redalyc/pdf/805/80501305. pdf> Accesado en: 8 feb. 2012.

INGENIEROS, J. La psicología en la República Argentina. Anales de Psicología. Sociedad de Psicología de Buenos Aires, I. 1910, p. 341. En: VEZZETTI $H$. (Comp.) El nacimiento de la psicología en la Argentina. Pensamiento psicológico y positivismo. Buenos Aires: Sudamérica, 1996, p. 55-60.

Los estudios psicológicos en la Argentina. Revista de Filosofía, Cultura, Ciencias, Educación. V. 1919. pp. 296. En: VEZZETTI H. (Comp.) El nacimiento de la psicología en la Argentina. Pensamiento psicológico y positivismo. Buenos Aires: Sudamérica, 1996, p. 61-78.

KLAPPENBACH, $\mathrm{H}$. Los orígenes de la psicología en Argentina. En PANTANO C., D. Inicios de la psicología en Argentina. San Juan Argentina. Subsecretaria de Cultura. Ministerio de Desarrollo humano. Gobierno de la Provincia de San Juan, 1997, p. 103-190.

. El movimiento de la higiene mental y los orígenes de la Liga Argentina de Higiene Mental. Temas de historia de la psiquiatría Argentina, Buenos Aires, n. 10, 1999.

. Dos editoriales en los comienzos de la profesionalización de la psicología en Argentina. Memorándum, Belo Horizonte, v.1, p. 61-71, 2001. Retirado em: <http://www.fafich.ufmg.br/ memorandum/artigos01/klappenbach0 1.htm>. Accesado en:I 8 feb. 2012.

Recepción de la Psicología Alemana y Francesa en la Temprana Psicología Argentina. Mnemosine, Río de Janeiro, v.2, n. 1, p. 75-86, 2006‥

Periodización de la psicología en Argentina, Revista de Historia de la Psicología, Valencia, v.27, n. 1, p. 109-164, 2006 b. Filosofía y política en el primer congreso Argentino de filosofía. Fundamentos en Humanidades, San Luís, enero-Junio, 
Año 1, n.1, p. 22-38, 2000. Disponible en: <http://redalyc. uaemex.mx> Accesado en: 12 feb. 2012.

- La psicología argentina al promediar el siglo XX: la figura de Luis María Ravagnan. Memorándum, Belo Horizonte, v.17, p. 74-87, 2009.

Disponible en_<http: //www.fafich.ufmg.br/ memorandum/a17/klappenbach02. pdf $>$ Accesado en: 2 en. 2011.

KLAPPENBACH, H.; PAVESI, P. Una historia de la psicología en Latinoamérica. Revista Latinoamericana de Psicología, Colombia, v.26, n.003, p. 445-481, 1994.

LIQUENO, J. M. Compendio de Psicología Contemporánea. De los anales de la Facultad de Derecho, Córdoba, Bautista Cubas, 1919.

MARSAL, A. La organización del laboratorio de fisiología experimental de la facultad de ciencias médicas de Córdoba. Folleto Academia Nacional de Ciencias, Córdoba, Miscelánea, n. 53, 1973.

MARTÍ NEZ, G. Programa de Semeiología. Archivo de la Facultad de Ciencias Médicas Universidad Nacional de Córdoba, 1925.

PAOLANTONIO, S.; MINNITI, E. Córdoba, Sarmiento, Gould y el mundo. Ponencia presentada en $\mathbf{V}$ Jornadas de Historia de Córdoba, 2004.

PAPINI, M. R. Datos para una historia de la psicología experimental Argentina (Hasta 1930). Revista Latinoamericana de Psicología, Bogotá, vol. 8, n. 2, p. 319-335, 1976. Dosponible en: <http: // redalyc.uaemex.mx/redalyc/pdf/805/80580212.pdf>

Accesado en: 3 feb. 2012.

PIÑEDA, M. A. La creación de la carrera de psicología en universidades católicas Argentinas. Memorándum, Belo Horizonte, 12,p.06-28,2007. Disponible

en:

<http://www.fafich. ufmg.br/ memorandum/a12/pineda03.htm>

Accesado en: 2 jul. 2009.

Comienzos de la profesionalización de la psicología, la Universidad Nacional de Córdoba y el movimiento neoescolástico. Memorándum, Belo Horizonte, v.7, p. 165-188, 2004. Disponible en: <http: //www. fafich. ufmg. br/ memorandum/artigos07/pineda01.h tm> Accesado en: 2 abr. 2005.

. El impacto de la Psicología Neoescolástica Experimental en Argentina, a través de libros de psicología de circulación en el país: 1935 - 1965. Memorándum, Belo Horizonte, v.8, p. 88-105, 2005, Disponible

en: <http://www.fafich. ufmg.br/ memorandum/artigos08/pineda02.htm $>$ Accesado en: 2 jul. 2007.

PLOTKIN, M. B. Freud en la Universidad de Buenos Aires: la primera etapa hasta la creación de la carrera de Psicología [en línea] EI Seminario, Buenos Aires. Disponible en: <http://www.elseminario.com.ar/biblioteca/Plotkin_Freud_Universida d> Accesado en: 3 nov. 2003. 
ROSSI, L. Historias clínicas y fichas, Criterios psicológicos implícitos según campos profesionales y contextos políticos. Argentina 19001957: Actas del IX Encuentro Argentino de Historia de la Psiquiatría, la Psicología y el Psicoanálisis. Buenos Aires, 3 y 4 de octubre. Edición digital. 2008.

. La psicología antes de la profesión. El desafío de ayer: instituir las prácticas. Buenos Aires: Eudeba. 1997.

ROSSI, L. El diseño de las publicaciones periódicas en Argentina. Actas de las XII Jornadas de Investigación y Primer Encuentro de Investigadores en Psicología del MERCOSUR. Buenos Aires, 4, 5 y 6 de agosto, 2005, p. 159-164.

. Publicaciones periódicas en Argentina: Producción discursiva e institucionalización. Referencias a la psicología. Anuario de Investigaciones, v. 14, Ciudad Autónoma de Buenos Aires. 2007 Disponible en: <http://www.scielo.org.ar/scielo.php?pid=S1851_168620070001000 46\&script=sci_arttext\&tIng=pt> Accesado en: 2 abr. 2010.

ROSSI, L., Ibarra, F. y Kirsch, U. Psicología en Argentina: Criterios psicológicos en los diseños de fichas del área laboral (1920-1945), Anuario de Investigaciones, Facultad de Psicología, UBA (XVII), 401-407, $2010 . \quad$ Disponible

en: <http://www.scielo.org.ar/pdf/anuinv/v17/v17a41.pdf> Accesado en: 10 jul. 2012

SCHERMAN, P. De las prácticas experimentales de la psicología a la profesión de psicólogo. Universidad Nacional de Córdoba 1900-1960. 2009. 431f. Tesis (Doctorado en Psicología). Universidad Nacional de Córdoba, 2009.

S/D. Retrato de Virgilio Ducceschi. U.S. National Library of Medicine, History of Medicine Division. De I mages from the history of medicine (IHM) Disponible en: <http://ihm.nlm.nih.gov/luna/servlet/detail/NLMNLM 1 1 1014139 72 174073: Prof--Virgilio-Ducceschi\#> S/F Accesado en: 15 mar. 2012.

TALAK, A. M. Psicología, fisiología y patología en los primeros desarrollos académicos de la psicología en la Argentina (1896-1919). Presentado al I Congreso I beroamericano de Filosofía de la ciencia y la Tecnología. Morelia, México 25 al 29 de Set. 2000a. Disponible en: <http://www.elseminario.com.ar> Accesado en 23 ago. 2007.

La psicología evolutiva en los primeros desarrollos de la psicología en la Argentina. Trabajo presentado en las VII J ornadas de I nvestigación. Facultad de Psicología. Universidad de Buenos Aires, Argentina. 24 y 25 de agosto. 2000b. Disponible en: <http://www.elseminario.com.ar/biblioteca/Talak_Psicologia_evolutiv a.htm> Accesado en: 4 ago. 2009. 
psicología social del positivismo. La articulación de discursos psicológicos y discursos ético-políticos en el pensamiento positivista sobre lo social. Programa de Estudios Históricos de la Psicología en la Argentina. Facultad de Psicología. UBA. Ponencia presentada en las VI Jornadas de Investigación en Psicología, Facultad de Psicología, Universidad de Buenos Aires, 2 y 3 de diciembre, 1999.1 Disponible en: <http://www.elseminario.com.ar/biblioteca/Talak_Psicologia_social_p ositivismo.htm> Accesado en: 23 sep. 2009.

VEZZETTI, H. La locura en la argentina. Buenos Aires: Paidós, 1985.

Estudios históricos de la psicología en Argentina. Cuadernos Argentinos de Historia de la Psicología, Facultad de Ciencias Humanas, Universidad Nacional de San Luis, San Luís, v. 2, n. 1/2, 1996a.

EI nacimiento de la psicología en la Argentina. Pensamiento psicológico y positivismo. Buenos Aires: Sudamérica. 1996b.

.Freud en Buenos Aires. Buenos Aires. Universidad Nacional de Quilmes. Segunda edición ampliada. 1996.

Aventuras de Freud en el país de los argentinos. Buenos Aires, Paidós, 1996c.

. Applied Psychology in Argentina: "psycho-hygiene" in the early days of its profesionalization. Presentado en el $\mathbf{2 4}^{\text {th }}$ International Congress of Applied Psychology, San Francisco, del 9 al 14 de agosto de 1998.

. Historia del freudismo e historia de la sexualidad: el género sexológico en Buenos Aires en los treinta, Prismas. Revista de historia intelectual, Universidad Nacional de Quilmes, Buenos Aires, n. 1, p.221-218, $1997 . \quad$ Disponible en: <http://saavedrafajardo.um.es/WEB/archivos/Prismas/01/Prismas0126. pdf > Accesado en: 17 jul. 2008.

\section{Endereço para correspondencia}

Fernando J osé Ferrari

Dirección: Enfermera Gordillo esquina Enrique Barros, Ciudad Universitaria. Córdoba, Argentina, C.P. 5000

Correo electrónico: ferrarijfernando@gmail.com

Recebido em: 23/03/2012

Reformulado em: 06/12/2012

Aceito para publicação em: 10/12/2012

Acompanhamento do processo editorial: Ana Maria Jacó Vilela

Notas

* Prof. asistente en la cátedra de Escuelas Corrientes y Sistemas de la Psicología Contemporánea de la Facultad de Psicología de la Universidad Nacional de Córdoba, 
Doctor en Psicología, investigador de la Secretaría de Ciencia y Técnica (SeCyT), Investigador del Centro de Estudios Avanzados (CEA). 